\title{
Characterization of small populations with microsatellite markers on mouse chromosome 11
}

\author{
Gudrun A. BROCKMANN* and Martina LANGHAMMER
}

\begin{abstract}
Brockmann G. A. and Langhammer M. 1998. Characterization of small populations with microsatellite markers on mouse chromosome 11. [In: Ecological genetics in mammals III. G. B. Hartl and J. Markowski, eds]. Acta Theriologica, Suppl. 5: 171-177.

Microsatellites of mouse chromosome 11 were used to characterize genomic changes on this chromosome in small populations. Populations were kept under different mating principles to (1) minimize the rate of inbreeding (MRI) and (2) to conserve the phenotypes of the basic population (BPC). Both populations were generated from a common base population with an effective population size of 20 mating pairs. After 13 generations the phenotypic values of body weight did not differ significantly from the initial population in both mating variants. However, the estimated coefficient of inbreeding was significantly increased in variant BPC compared to MRI. Microsatellite marker analysis proved the occurence of significant differences in allele frequency distributions between both mating variants as recorded at the 13th generation. Significantly, a complete loss of specific microsatellite alleles was evident in both mating variants. The number of lost alleles in the variant BPC was twice as high as in the variant MRI. Thus, microsatellite analyses confirmed the expectation that the consequent reduction of inbreeding contributes to both reduction of genetic losses and maintenance of the original phenotypes.
\end{abstract}

Research Institute for the Biology of Farm Animals, 18196 Dummerstorf, Wilhelm-Stahl-Allee 2, Germany

Key words: small populations, body weight, genetic markers, mouse, inbreeding

\section{Introduction}

In domestic animals as well as in zoo and wild animals the preservation of genetic variety is of major importance to keep a wide scale of different species, races, and lines in our colorful nature. This natural abundance is, on the one hand, prerequisite for the adaptability to changing environmental conditions, and, on the other hand, it provides the genetic resources in animal breeding for selection of different and changing traits from among vital farm animals. Traditional methods of selection combined with artificial insemination and embryo transfer are prone to cause losses of genetic variability. Furthermore, the use of a reduced number of favorite breeding bulls in large populations contributes in traditional

\footnotetext{
*Corresponding address: Forschungsinstitut für die Biologie landwirtschaftlicher Nutztiere, FB Molekularbiologie, Wilhelm-Stahl-Allee 2, 18196 Dummerstorf, Germany, fax: 049-38208 68702, e-mail: brockman@fbn-dummerstorf.de
} 
breeding strategies to a quick dissemination of favorable genomic variants resulting in the improvement of the trait. Yet, recessive inherited negative effects of secondary traits or even defect genes may cosegregate with the same speed. Thus, it is a general aim of breeders to include new genetic information into selection programs to increase the precision of the selection response on the background of highly viable animals. Depending upon selection requirements, it may be necessary to include the genetic determinants of various traits being conserved in small populations into selection strategies. In livestock production numerous endangered populations are kept to maintain their typical characteristics, while inbreeding is minimized. Guidelines for conservation by living animals are given by Frankham 1995. These include the maximization of initial heterozygosity, the minimization of the number of generations, and the maximization of the effective population size.

In our model study small mouse populations which have been kept under different mating schemes were analyzed for their genetic variability. The mating principles choosen served to (1) minimize the rate of inbreeding in the MRI population and (2) to conserve the original phenotypes of the basic population in the BPC population. Exploiting this material, our study seeks to evaluate changes in the phenotypic trait, the development of the inbreeding coefficients, and to analyze allele frequencies of genetic markers at the beginning of the experiment and after 13 generations. Body weight at 42 days was recorded as phenotypic trait. Microsatellite markers of mouse chromosome 11 were used to assess changes of genetic diversity within both mating variants. A linkage of markers on this chromosome with a quantitative trait locus (QTL) affecting body weight was proven in the initial base population (Brockmann et al. 1996). Both mating variants might yield different changes in allele frequencies of genetic markers linked with the QTL.

\section{Material and methods}

We used as stock population the outbred strain Fzt:Du which was generated in 1969 by systematic crossbreeding of four inbred and four outbred strains (Schüler 1985). At the beginning of the experiment full-sibs of 42 different pubs of this strain were distributed to the two small populations MRI and BPC. Starting with the first generation the effective population size was fixed at 20 males and 20 females. Animals were mated at a ratio of 1:1. The two small populations differed in their mating schemes. Computer programs were developed for two different mating principles which provided a mating regime (1) to minimize the rate of inbreeding (MRI) in one population, and (2) to maintain the original phenotypic distribution of body weight of the basic population (BPC) in the other population. In the mating variant MRI animals with lowest kinship coefficients estimated throughout the following three generations were mated without consideration of phenotypic traits. In the variant BPC those animals with lowest differences between the pedigree value for body weight and the breeding value of the basic animals were used for the mating decision. Full-sib mating was not allowed in both populations. The following model was used for breeding value estimation:

$y_{i j k l m}=\alpha_{i j}+\beta_{k}+\gamma_{k}+w_{i j l}+u_{i j l m}+e_{i j k l m}$, with $\alpha$-effect of generation, $\beta$ - effect of repeat, $\gamma=$ effect of $\mathrm{sex}, \mathrm{w}=$ effect of litter, $\mathrm{u}=$ effect of animal, $\mathrm{e}=$ residual. The pedigrees were used for genetic analyses 
and the estimation of inbreeding coefficients. For the comparison of allele frequency changes the $\chi^{2}$-test was applied (Rasch et al. 1978).

The body weight at 42 days was recorded for each animal as the phenotypic trait. Animals were fed ad libitum.

Microsatellites D11Mit236, D11Mit36, D11Mit213, D11Mit125, D11Mit126, D11Mit180 and D11Mit214 (Research Genetics, Huntsville) were used as genetic markers to characterize genetic diversity of the small populations. All animals of both populations were genotyped at the beginning of the experiment and after 13 generations. DNA for microsatellite analyses was prepared from mouse tails clips. PCR and fragment analyses were carried out as described previously (Das et al. 1996). A genotype standard was loaded for each microsatellite locus on all gels to assure proper data analyses.

\section{Results}

\section{Body weight}

Data on the body weights at day 42 of the mating variants minimal inbreeding rate (MRI) and basic phenotype conservation (BPC) are presented in Table 1 . In

Table 1. Mean body weights and standard errors (SE) for the populations MRI (minimal rate of inbreeding) and BPC (basic population conservation) at generations 0 and 13 . ${ }^{\mathrm{a}, \mathrm{b}}$ - significantly different at $p<0.05$.

\begin{tabular}{lccllll}
\hline & \multicolumn{2}{c}{ Generation 0} & & \multicolumn{2}{c}{ Generation 13} \\
\cline { 2 - 3 } \cline { 5 - 6 } & Mean & SE & & Mean & SE \\
\hline MRI & 27.91 & 3.18 & & $28.73^{\mathrm{a}}$ & 3.37 \\
BPC & 27.58 & 4.23 & & $27.59^{\mathrm{b}}$ & 2.84 \\
\hline
\end{tabular}

variant MRI the phenotype was not considered for the mating scheme, while the mating scheme in variant BPC should preserve the phenotype of the base population. Initially, the body weight was alike in both experimental groups, since they were descendant from sibpairs of the same base population. No significant difference in body weight was evident until generation 13 within each population compared with generation 0 , and between populations at the same generation.

\section{Inbreeding}

Changes in the development of the coefficient of inbreeding was estimated for each small population via the kinship matrix of the population. In variant MRI inbreeding was minimized by mating only of those animals having the lowest kinship coefficients. These were estimated for the following three generations. The estimates for the inbreeding coefficients are shown in Table 2. Inbreeding increased in both lines. However, after 13 generations the inbreeding coefficient in the variant $\mathrm{BPC}$ was 2.6 times higher compared to MRI. The inbreeding rate 
Table 2. Estimates for the inbreeding coefficients and the gain of inbreeding calculated per generation in the populations MRI (minimal rate of inbreeding) and BPC (basic population conservation). In the population MRI animals with lowest kinship coefficients estimated for the following three generations were mated. In the population BPC only those animals were mated with lowest differences between the actual pedigree value for body weight and the breeding value of the basic population; full-sib matings were prohibited.

\begin{tabular}{lcc}
\hline & Inbreeding coefficients & Gain of inbreeding $(\%)$ \\
\hline MRI & 0.07 & 0.5 \\
BPC & 0.18 & 1.4 \\
\hline
\end{tabular}

per generation was accelerated by $1.4 \%$ in the BPC protocol, as opposed to cnly $0.5 \%$ under the MRI regime.

\section{Allele frequencies}

The development of both small populations was accompanied by the analyses of seven genetic markers located on mouse chromosome 11. These markers cover the entire chromosome, with an average distance of $10 \mathrm{~cm}$ between adjacent markers. In the base population, 3 to 6 different microsatellite alleles per marker were detected. Chi-square testing was applied to compare shifts in allele frequencies between variants. Initially, both populations had similar frequencies of microsatellite alleles at all loci. Significant alterations of the allele frequencies were recorded after 13 generations in both mating variants. Six out of seven

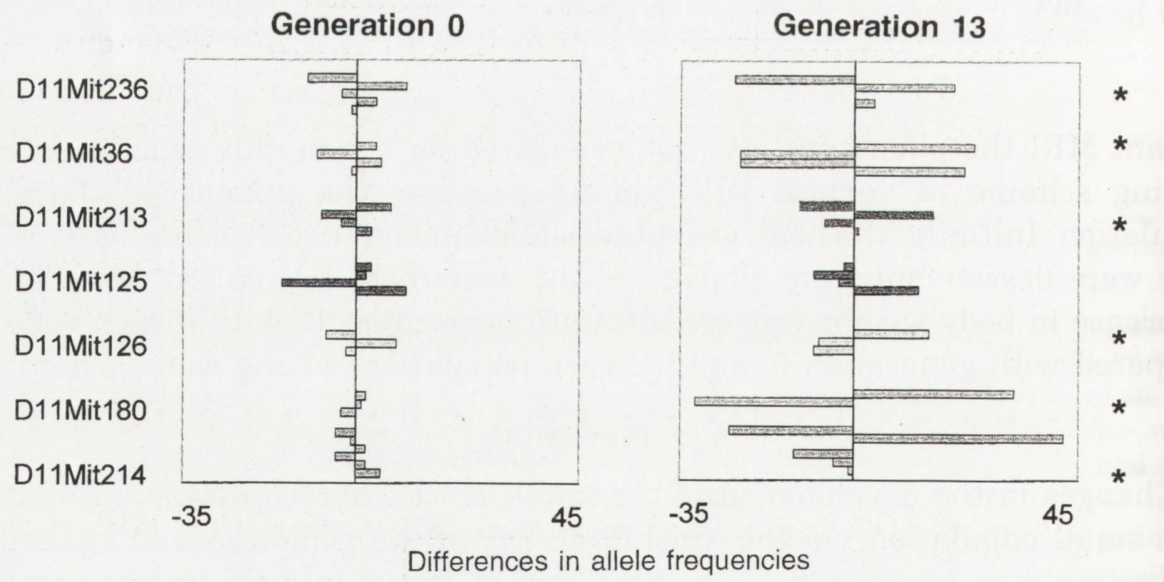

Fig. 1. Differences in the number of microsatellite alleles between the mating variants MRI and BPC in generations 0 and 13. The columns for each allele represent the difference of the absolute number of the allele in the population MRI versus BPC. In correspondence with the population size 82 alleles were present at each locus in generation 0 and 80 in generation 13. Significant allele frequency changes between both populations are labeled with asterisk $(p<0.05)$. 


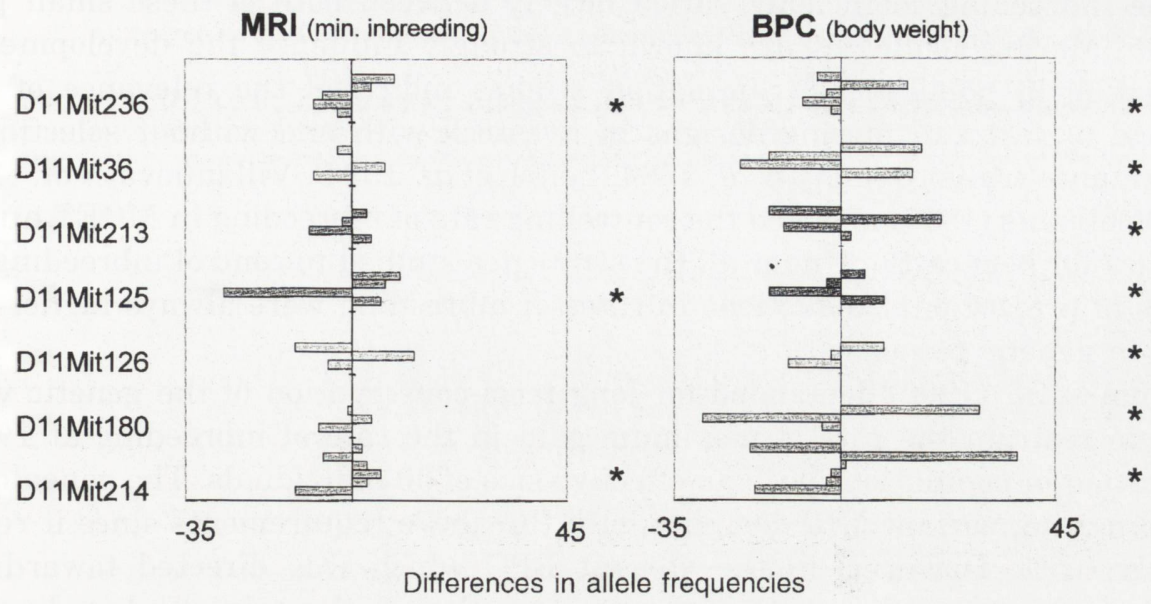

Fig. 2. Differences in the number of microsatellite alleles between the beginning of the experiment and after 13 generations are shown for both mating variants MRI and BPC. Significant allele frequency changes within a population after 13 generations are labeled with asterix $(p<0.05)$.

analyzed microsatellite markers showed significant changes between the MRI and BPC mating protocols. No difference was evident at locus D11Mit125 being located on the distal region of this chromosome. The differences in the allele distribution patterns between both small populations are shown in Fig. 1 for all markers at generations 0 and 13 .

Changes in allele frequency distributions within each population are summarized in Fig. 2. Within the variant BPC differences in allele frequencies between generations 0 and 13 were proved at all loci, while the allele frequencies of only three out of seven loci were influenced in variant MRI. In part, these changes in allele frequencies were accompanied by a complete loss of microsatellite alleles. From among 29 alleles having originally been in both populations, 6 and 3 alleles were lost in variants BPC and MRI, respectively, after 13 matings. Losses were highest at loci with a high number of alleles, but it was also observed at loci with only 3 alleles.

\section{Discussion}

The two small populations having been kept under different mating principles were analyzed for their phenotypic and genetic development over 13 generations. Both mating principles are suitable to maintain the phenotype of the base population with respect to body weight performance. Using a genealogical aimed mating design (MRI) and a phenotypic one (BPC) the body weights did not change significantly from the basic population after 13 generations. 
The inbreeding coefficients varied largely between both of these small populations. Mating strategies are known to strongly influence the development of inbreeding in populations. Simulation studies indicated the relevance of hierarchical or factorial mating designs in livestock with and without selection for performance traits (Grundy et al. 1994, Leitch et al. 1994). Villanueva et al. (1994) and Woolliams (1989) modeled the controlling rate of inbreeding in MOET nucleus schemes for beef cattle. Under all the strategies studied to control inbreeding, the effects of proportional reductions in rates of inbreeding were always higher than those in genetic response.

Brem et al. (1990) demanded for long term-conservation of the genetic variability to restrain the rate of maximum gain in the rate of inbreeding at $1 \%$ per generation in populations with an effective size of 50 individuals. The genealogical aimed mating variant MRI complies with the above requirements since it results in only $0.5 \%$. However, in the variant BPC which was directed towards the maintenance of the phenotype of the base population, the gain of inbreeding rate was $1.4 \%$. Thus, in this population the genetic variability can not be preserved to the same extent.

The analyses of microsatellites on chromosome 11 support this suggestion. The loss of alleles in variant BPC was twice as high as in variant MRI. Hence, only $10 \%$ of alleles were lost, if the kinship matrix was included into the mating decision. However, a loss of $20 \%$ was evident, if the phenotype was considered as exclusion criterion for the mating decision. The high proportion of significant differences in allele distribution patterns between the initial and the 13th generation combined with a high loss of marker alleles in the variant BPC is conceivably caused by the phenotype selection protocol applied for the mating decision. On chromosome 11, a quantitative trait locus affecting body weight was localized in segregating family populations from crosses of a mouse line selected for high body weight to a control line (Brockmann et al. 1996, 1998). The selected and control lines used for those crosses, and also the small populations examined here were established from the same basic population Fzt:Du. It may be assumed, that this population comprising 200 mating pairs preserves the highest number of QTL alleles. Probably, those alleles were enriched in the small population BPC contributing to an average body weight, while alleles responsible for extreme growth rates were lost. In small mouse populations selected divergently for high and low body weight from a common base population, an enrichment of specific marker alleles results from selection forces and genetic drift (Keightley et al. 1996). Therefore, the consequent reduction of inbreeding proves as superior strategy for the preservation of all alleles contributing to QTLs over mating of phenotypically highly selected animals.

Although the changes of allele frequencies were recorded only for microsatellites on chromosome 11, they might be representative for the entire genome.

Acknowledgement: Excellent technical assistance was provided by Hannelore Tychsen for DNA preparation and genotyping. 


\section{References}

Brem G., Brenig B., Müller M., Springmann K. and Kräußlich H. 1990. Genetische Vielfalt von Rinderrassen. Eugen Ulmer, Stuttgart: 1-6.

Brockmann G., Haley C. S., Renne U., Knott S. A. and Schwerin M. 1998. Quantitative trait loci affacting body weight and tatness from a house line selected for extreme high growth. Genetics 150: $169-181$.

Brockmann G., Timtchenko D., Das P., Renne U., Freyer G., Kuhla S., Teuscher F., Wolf J., Kühn C. and Schwerin M. 1996. Detection of QTL for body weight and body fat content in mice using genetic markers. Journal of Animal Breeding Genetics 113: 373-379.

Das P., Meyer L., Seyfert M., Brockmann G. and Schwerin M. 1996. Structure of growth hormone-encoding gene and its promoter in mice. Gene 169: 209-213.

Frankham R. 1995. Proccedings of the 5th World Congress of Genetics of Applied Livestock Production 21: 385-392.

Grundy B., Caballero A., Santiago E. and Hill W. G. 1994. A note on using biased parameter values and non-random mating to reduce rates of inbreeding in selection programmes. Animal Production 59: 465-468.

Keightley P. D., Hardge T., May L. and Bulfield G. 1996. A genetic map of quantitative trait loci for body weight in the mouse. Genetics 142: 227-235.

Leitch H. W., Smith C., Burnside E. B. and Quinton M. 1994. Genetic response and inbreeding with different selection methods and mating designs for nucleus breeding programs of dairy cattle. Journal of Dairy Science 77: 1702-1718.

Rasch D., Herrendörfer G., Bock J. and Busch K. 1978. Verfahrensbibliothek Versuchsplanung und -auswertung, Band 2. Deutscher Landwirtschaftsverlag Berlin: 884.

Schüler L. 1985. Mouse strain Fzt: Du and its use as model in animal breeding research. Archiv für Tierzucht 28: 357-363.

Villanueva B., Woolliams J. A. and Simm G. 1994. Strategies for controlling rates of inbreeding in MOET nucleus schemes for beef cattle. Genetics, Selection, Evolution 26: 517-535.

Woolliams J. A. 1989. Modifications of MOET nucleus breeding schemes to imptove rates of genetic progress and decrease rates of inbreeding in dairy cattle. Animal Production 49: 1-14.

Received 10 June 1998, accepted 30 June 1998. 